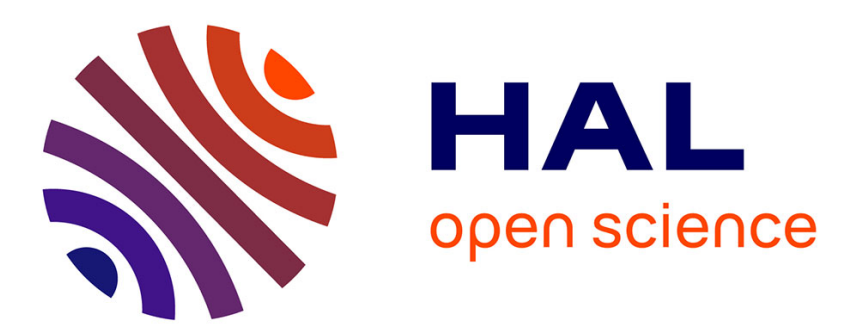

\title{
Restauration du frottement intérieur dans Mgo monocristallin déformé plastiquement a l'ambiante
}

\author{
M. Gabbay, C. Esnouf, Gilbert Fantozzi
}

\section{To cite this version:}

M. Gabbay, C. Esnouf, Gilbert Fantozzi. Restauration du frottement intérieur dans Mgo monocristallin déformé plastiquement a l'ambiante. Journal de Physique Lettres, 1978, 39 (15), pp.271-274. 10.1051/jphyslet:019780039015027100 . jpa-00231495

\section{HAL Id: jpa-00231495 https://hal.science/jpa-00231495}

Submitted on 1 Jan 1978

HAL is a multi-disciplinary open access archive for the deposit and dissemination of scientific research documents, whether they are published or not. The documents may come from teaching and research institutions in France or abroad, or from public or private research centers.
L'archive ouverte pluridisciplinaire HAL, est destinée au dépôt et à la diffusion de documents scientifiques de niveau recherche, publiés ou non, émanant des établissements d'enseignement et de recherche français ou étrangers, des laboratoires publics ou privés. 
Classification

Physics Abstracts 62.40

\title{
RESTAURATION DU FROTTEMENT INTÉRIEUR DANS MgO MONOCRISTALLIN DÉFORMÉ PLASTIQUEMENT A L'AMBIANTE
}

\author{
M. GABBAY, C. ESNOUF et G. FANTOZZI
}

Groupe d'Etudes de Métallurgie Physique et de Physique des Matériaux (*) I.N.S.A. Lyon, Bâtiment 502, 20, avenue Albert-Einstein, 69621 Villeurbanne Cedex, France

(Reçu le 8 mai 1978, révisé le 9 juin 1978, accepté le 13 juin 1978)

\begin{abstract}
Résumé. - La restauration du frottement intérieur est étudiée dans le domaine indépendant de l'amplitude $\left(\varepsilon_{\max }=10^{-7}\right)$. La déformation plastique fait croître simultanément le frottement intérieur entre 77 et $300 \mathrm{~K}$ et un pic $P_{1}$ situé vers $120 \mathrm{~K}$. Au cours du recuit, le pic $P_{1}$ est très stable alors que le frottement intérieur mesuré à $300 \mathrm{~K}$ décroît rapidement. Cette décroissance semble liée au recuit d'un pic $P_{2}$ situé vers $390 \mathrm{~K}$. La disparition des pics $P_{1}$ et $P_{2}$ se produit après chauffage à $770 \mathrm{~K}$ et $870 \mathrm{~K}$ respectivement, tandis que trois pics de faible hauteur apparaissent. La restauration complète est obtenue après chauffage à $970 \mathrm{~K}$. Un essai d'interprétation de ces résultats est proposé.
\end{abstract}

\begin{abstract}
Recovery of internal friction has been studied in the amplitude independent range $\left(\varepsilon_{\max }=10^{-7}\right)$. Plastic deformation gives rise to an increase of internal friction measured in the range $77 \mathrm{~K}-300 \mathrm{~K}$ and to a peak $P_{1}$ situated at about $120 \mathrm{~K}$. During annealing the $P_{1}$ peak is stable, whilst internal friction measured at $300 \mathrm{~K}$ decreases quickly. This decrease seems to be linked to the annealing of a peak $\left(P_{2}\right)$ located near $390 \mathrm{~K}$. Disappearance of the $P_{1}$ and $P_{2}$ peaks occurs after heating up to $770 \mathrm{~K}$ and $870 \mathrm{~K}$ respectively while three new small peaks appear. Total recovery is obtained after heating up to $970 \mathrm{~K}$. An interpretation of these results is suggested.
\end{abstract}

1. Introduction. - Le frottement intérieur a été fréquemment utilisé pour l'étude du comportement des dislocations dans les cristaux ioniques [1]. En ce qui concerne $\mathrm{MgO}$ [2-6], les résultats, obtenus dans des conditions d'expérimentation souvent très différentes, ne présentent pas de corrélations suffisantes pour permettre de dégager une idée générale claire sur les mécanismes qui contrôlent le mouvement des dislocations. Ceci est peut être dû au fait que le mode de déformation plastique utilisé est parfois mal défini (abrasion) et conduit à une importante dispersion des résultats expérimentaux. Il nous a donc semblé intéressant d'étudier le comportement anélastique de $\mathrm{MgO}$ après une déformation plastique effectuée dans des conditions les plus reproductibles possibles : nous avons choisi de déformer les monocristaux parallélépipédiques par compression selon le plus grand axe $\langle 100\rangle$ (bien plus long que les deux autres), ce mode de déformation ayant été largement étudié. De plus, les progrès réalisés dans la

(*) E.R.A. n 463. connaissance des défauts ponctuels et linéaires créés par déformation plastique nous aideront dans l'interprétation des résultats. Ainsi par exemple les études théoriques de Woo et Puls [7] et expérimentales de Singh et Coble [8] sur la mobilité des dislocations montrent que, pour les cristaux suffisamment purs, un mécanisme du type Peierls-Nabarro contrôle le mouvement des dislocations ; dans le cas des cristaux dopés, l'interaction dislocation-impuretés peut devenir prépondérante [8]. A la lumière de ces résultats, on peut espérer mettre en évidence après déformation plastique, des manifestations anélastiques caractéristiques des mécanismes précédents et apporter ainsi des informations complémentaires sur l'évolution des défauts.

2. Conditions expérimentales. - Les échantillons fournis par Norton Cie sont des parallélépipèdes de $40 \times 4 \times 2 \mathrm{~mm}^{3}$ taillés selon les faces de clivage (100). Les résultats de l'analyse chimique réalisée par le Centre d'Etudes de Chimie Métallurgique de Vitrysur-Seine sont présentés dans le tableau I 


\section{TABLEAU I}

en $\mu \mathrm{g} \cdot \mathrm{g}^{-1}$

$\begin{array}{ccccccccc}\mathrm{Fe} & \mathrm{Si} & \mathrm{Ca} & \mathrm{Al} & \mathrm{Ti} & \mathrm{Mn} & \mathrm{Cr} & \mathrm{Ni} & \mathrm{Zn} \\ \overline{90} & \overline{30} & \overline{30} & \overline{50} & \frac{1}{12} & \overline{8} & \overline{8} & \overline{5} & \overline{5}\end{array}$

Avant déformation plastique, chaque échantillon subit le traitement suivant :

- un polissage chimique : dans l'acide $\mathrm{H}_{3} \mathrm{PO}_{4}$ à $100{ }^{\circ} \mathrm{C}$ pour éliminer les défauts de surface ;

- un traitement thermique : chauffage à l'air à $1400{ }^{\circ} \mathrm{C}$ pendant 48 heures pour stabiliser les impuretés fer ou chrome dans l'état trivalent puis refroidissement rapide à l'air $(<3 \mathrm{~min})$ pour conserver une distribution uniforme des impuretés ;

- une métallisation pour permettre l'excitation électrostatique des vibrations transversales.

Un dispositif électronique automatique permet de réaliser la mesure du décrément logarithmique $\delta$, de la fréquence de vibration (de l'ordre de $10 \mathrm{kHz}$ ) en fonction de la température $(77 \mathrm{~K}<\mathrm{T}<673 \mathrm{~K})$ au cours d'une montée linéaire de $40 \mathrm{~K} \mathrm{~h}^{-1}$. L'amplitude maximale de vibration $\varepsilon_{\max }$ est maintenue

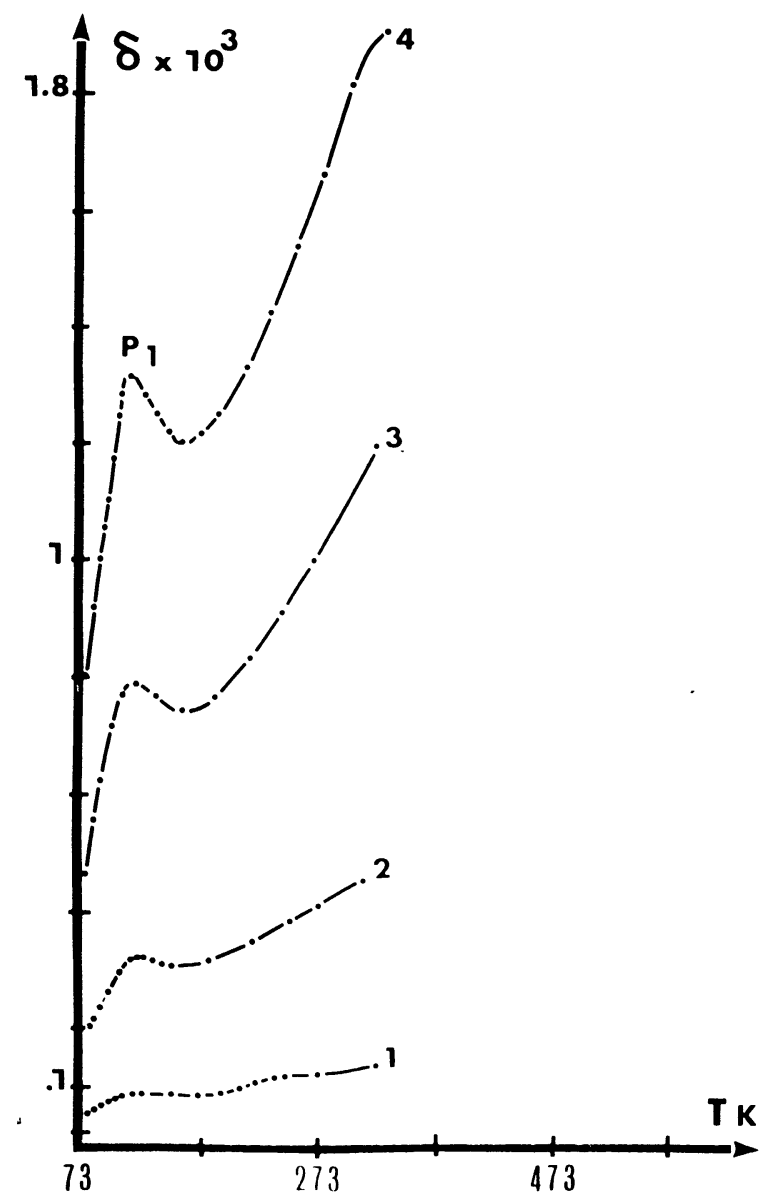

FIG. 1. - Effet du taux de déformation plastique sur les courbes $\delta(T): 1 . \varepsilon_{\mathrm{p}}=0,4 \% ;$ 2. $\varepsilon_{\mathrm{p}}=1 \%$; 3. $\varepsilon_{\mathrm{p}}=2 \%$;

[Effect of plastic deformation on $\delta(T)$ curves : 1, $\varepsilon_{\mathrm{p}}=0.4 \%$ 2. $\varepsilon_{\mathrm{p}}=1 \% ; 3 . \varepsilon_{\mathrm{p}}=2 \% ; 4 . \varepsilon_{\mathrm{p}}=3.4 \%$.] constante à $\varepsilon_{\max }=10^{-7}(\delta$ est alors indépendant de l'amplitude). Les monocristaux sont déformés plastiquement en compression selon le plus grand axe $\langle 100\rangle$, à l'aide d'une machine Instron, la vitesse de déformation étant de l'ordre de $2 \times 10^{-6} \mathrm{~s}^{-1}$. Théoriquement quatre plans de glissement (110) $\langle 1 \overline{1} 0\rangle$ sont identiquement sollicités, mais pratiquement deux seulement sont effectivement activés dans des proportions inégales.

3. Résultats expérimentaux. - EFFET DE LA DÉFORMATION PLASTIQUE. - La figure 1 montre l'évolution du spectre $\delta(T)$ entre $77 \mathrm{~K}$ et $293 \mathrm{~K}$ avec le taux de déformation plastique $\varepsilon_{\mathrm{p}}$. Le frottement intérieur croît considérablement avec $\varepsilon_{\mathrm{p}}$, un pic $\mathrm{P}_{1}$ vers $120 \mathrm{~K}$ se développant simultanément.

EFFET DU RECUIT. - L'évolution générale des courbes $\delta(T)$ en fonction du recuit après déformation plastique de $3,4 \%$ est représentée sur les figures 2 et 3. L'effet du recuit sur le frottement intérieur se manifeste immédiatement au-dessus de l'ambiante. Le frottement intérieur diminue rapidement (Fig. 2 courbes 5-9) ; il apparaît un léger épaulement situé vers $280 \mathrm{~K}$ et après recuit vers $500 \mathrm{~K}$, un pic bien défini situé vers $390 \mathrm{~K}\left(\mathrm{P}_{2}\right)$ (Fig. 2 courbes 8 et 9 ).

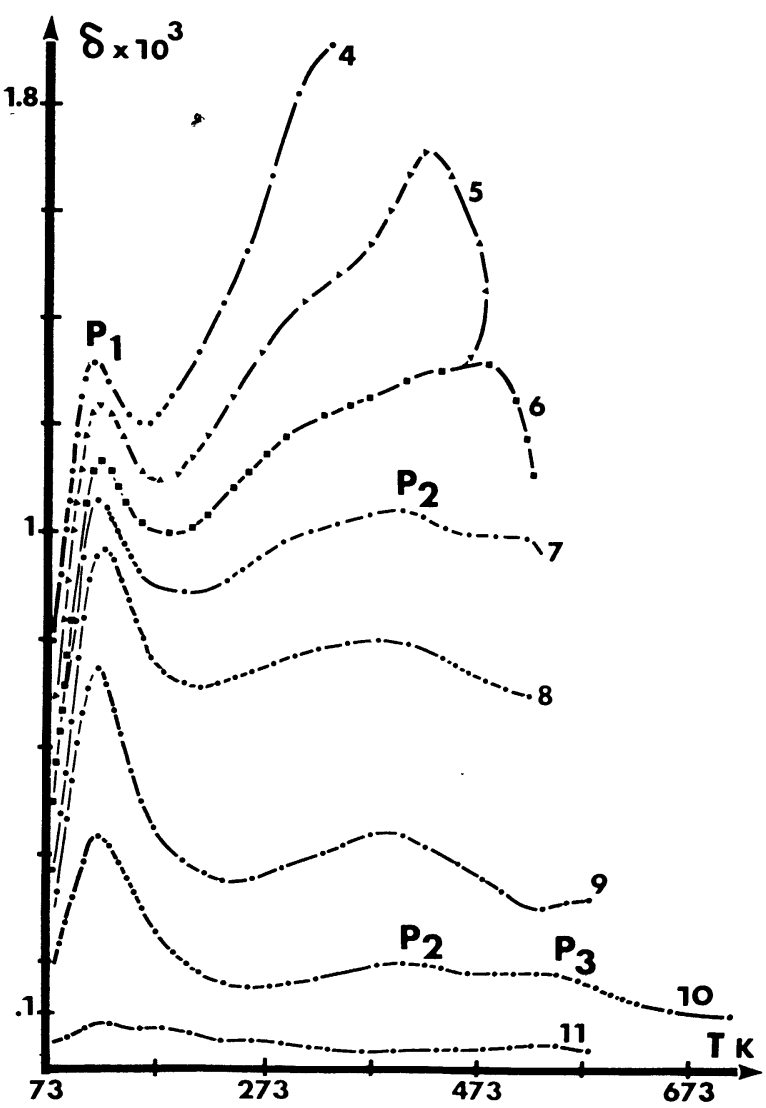

Fig. 2. - Effet du recuit sur les courbes $\delta(T)$ : 4. après déformation, 5. après recuit à $408 \mathrm{~K} ; 6.478 \mathrm{~K} ; 7.523 \mathrm{~K} ; 8.529 \mathrm{~K}$; 9. $675 \mathrm{~K} ; 10.783 \mathrm{~K} ; 11.893 \mathrm{~K}$.

[Effect of annealing on $\delta(T)$ curves : 4. after deformation ; 5. after annealing at $408 \mathrm{~K} ; 6.478 \mathrm{~K} ; 7.523 \mathrm{~K} ; 8.529 \mathrm{~K} ; 9.675 \mathrm{~K}$; 10. $783 \mathrm{~K} ; 11.893 \mathrm{~K}$. 
Ce pic $\mathrm{P}_{2}$ disparaît presque complètement après recuit à $783 \mathrm{~K}$ tandis qu'un nouveau petit pic $\mathrm{P}_{3}$ apparaît vers $530 \mathrm{~K}$ (Fig. 3 courbe 10). Un chauffage à $976 \mathrm{~K}$ élimine toutes les manifestations anélastiques précédentes (Fig. 3 courbe 12)

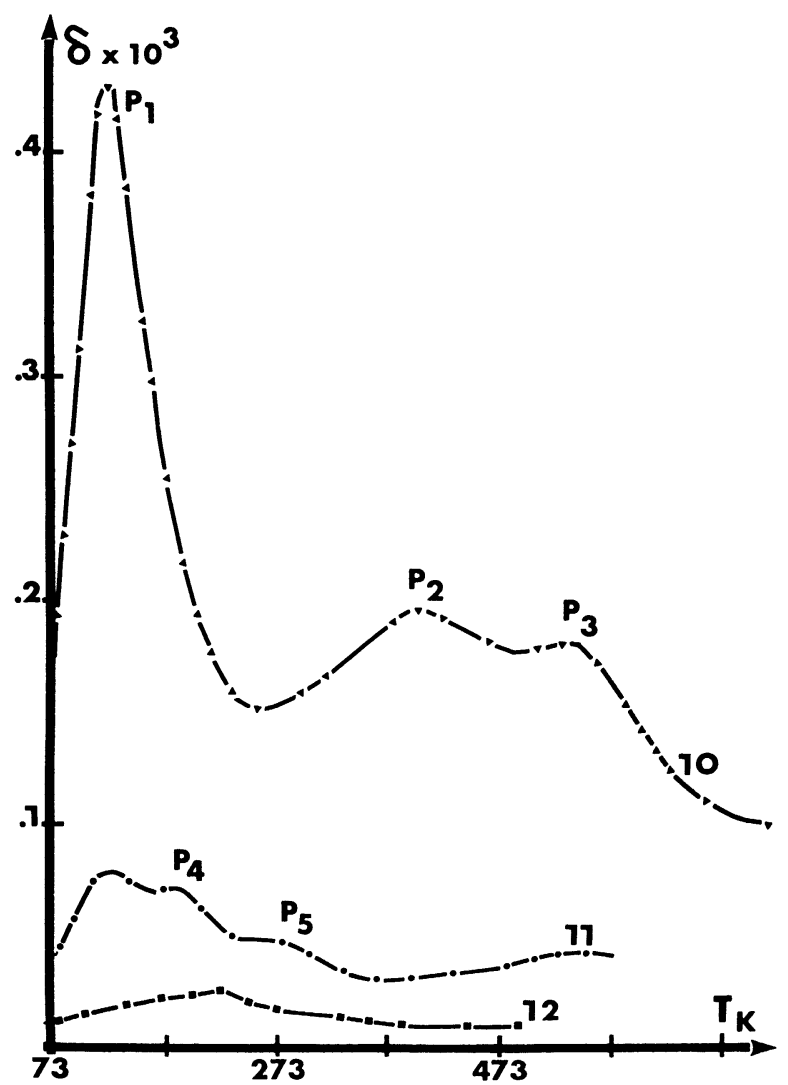

FIG. 3. - Effet du recuit sur les courbes $\delta(T)$, les échelles sont dilatées par rapport à la figure $2: 10$. après recuit à $783 \mathrm{~K}$; 11. $893 \mathrm{~K} ; 12.976 \mathrm{~K}$.

[Effect of annealing on $\delta(T)$ curves, $\delta$ scale is expanded in comparison with fig. 2 : 10. after annealing at $783 \mathrm{~K} ; 11.893 \mathrm{~K}$; 12. $976 \mathrm{~K}$.

Le pic $P_{1}$ mis en évidence immédiatement après déformation plastique est stable lors du recuit jusqu'à $780 \mathrm{~K}$ (Fig. 2), sa forme s'affinant avec la baisse du frottement intérieur (Figs 2, 3). Après chauffage à $890 \mathrm{~K}$, il est presque totalement recuit, deux nouveaux pics $\mathrm{P}_{4}(\mathrm{à} 180 \mathrm{~K})$ et $\mathrm{P}_{5}(\mathrm{à} 280 \mathrm{~K})$ de faible hauteur apparaissant (Fig. 3 courbe 11).

4. Discussion. - L'élévation du frottement intérieur avec le taux de déformation plastique est due à l'augmentation de la densité des dislocations mobiles introduites par cette déformation. Nous venons de voir que le spectre de frottement intérieur est essentiellement constitué par les deux pics $P_{1}$ et $P_{2}$ et par trois autres pics $P_{3}, P_{4}, P_{5}$ de hauteurs bien plus faibles. Examinons successivement ces différentes manifestations anélastiques.

$\mathrm{Au}$ sujet du pic $\mathrm{P}_{1}$, ses caractéristiques principales sont les suivantes :

i) la hauteur de $P_{1}$ croît avec le taux de défor-

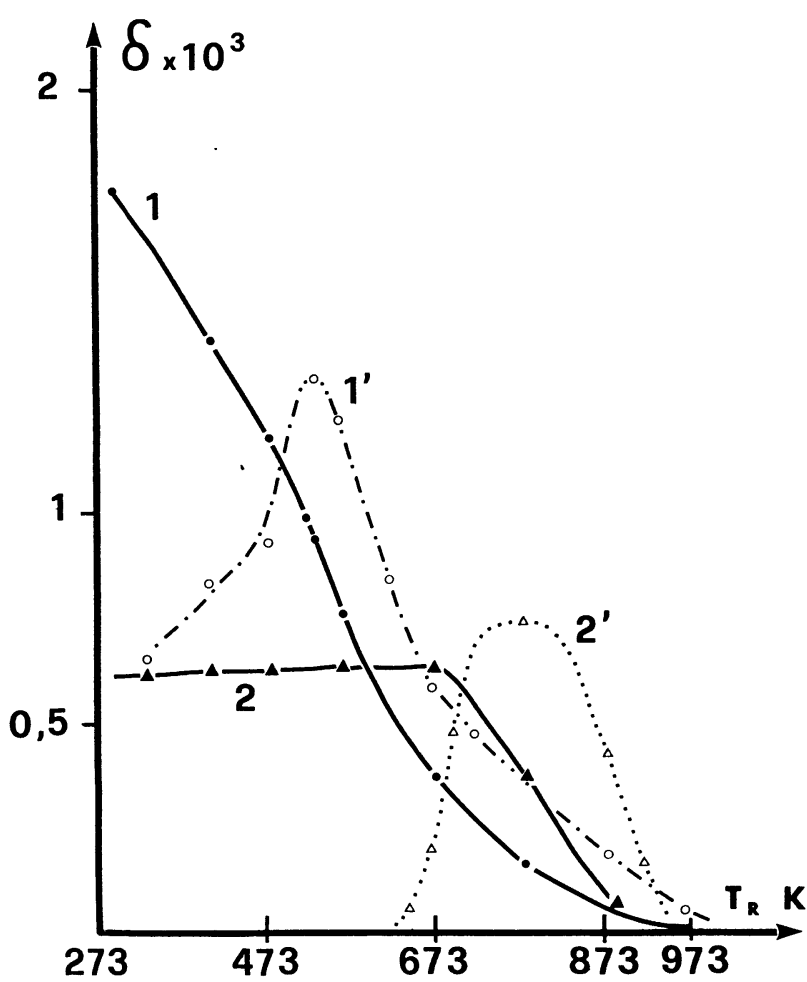

FIG. 4. - Variation du frottement intérieur mesuré à $293 \mathrm{~K}$ (courbe 1) et de la hauteur du pic $\mathrm{P}_{1}$ (courbe 2) en fonction de la température de recuit $T_{\mathrm{R}}$ et courbes dérivées ( $1^{\prime}$ et $2^{\prime}$ ).

[Change of internal friction measured at $293 \mathrm{~K}$ (curve 1) and of $\mathrm{P}_{1}$ peak height (curve 2) as a function of annealing temperature $T_{\mathrm{R}}$ and derived curves $\left(1^{\prime}\right.$ and $\left.2^{\prime}\right)$.]

mation plastique $\varepsilon_{\mathrm{p}}$ (Fig. 1) et demeure quasi constante en fonction de la température de recuit jusqu'à $670 \mathrm{~K}$; elle décroît ensuite entre $670 \mathrm{~K}$ et $870 \mathrm{~K}$ (Fig. 4 , courbes 2 et $2^{\prime}$ ). (La hauteur de $P_{1}$ a été estimée par décomposition du spectre global de frottement intérieur) ;

ii) la hauteur de $P_{1}$ n'est pas sensible à l'amplitude de vibration (jusqu'à $\varepsilon_{\max }=10^{-5}$ );

iii) la température de $P_{1}$ n'est pas affectée par le taux de déformation, le recuit et l'amplitude de vibration ;

iv) le pic $P_{1}$ présente un facteur d'élargissement de l'ordre de 3 à 4 (rapport largeur à mi-hauteur du pic $P_{1}$ sur largeur d'un pic de Debye).

Ces caractéristiques permettent de penser que le pic $P_{1}$ est lié à une propriété intrinsèque des dislocations. Ce pic peut être associé aux pics observés par Chang [5] et Ikushima et Suzuki [6] vers $270 \mathrm{~K}$ à $50 \mathrm{MHz}$. L'énergie d'activation $E$ et la fréquence d'attaque $f_{0}$ déduites de l'ensemble des résultats sont :

$$
E=(0,16 \pm 0,03) \mathrm{eV} \quad 10^{10}<f_{0}<10^{11} \mathrm{~s}^{-1}
$$

Cette énergie d'activation est plus faible que l'énergie de création des doubles décrochements mesurée expérimentalement par Singh et Coble [8] et théorique- 
ment par Woo et Puls [7] (égales à 0,5 et $0,8 \mathrm{eV}$ pour les dislocations coins et vis respectivement). En conséquence, on ne peut identifier le pic $\mathrm{P}_{1}$ à un pic de Bordoni et il est raisonnable de l'attribuer au mouvement latéral des décrochements géométriques.

Dans le domaine de température de recuit du pic $\mathrm{P}_{1}$, les études d'absorption optique montrent que les défauts ponctuels introduits par déformation plastique évoluent $[9,10]$. L'identification des défauts fait encore l'objet d'hypothèses diverses mais il semble que l'épinglage des décrochements géométriques puisse être attribué au recuit des centres $\varepsilon$, probablement accompagné d'une migration de lacunes cationiques.

Le pic $P_{2}$, situé vers $390 \mathrm{~K}$, n'apparaît nettement qu'après recuit vers $480 \mathrm{~K}$ et il est par conséquent difficile de dire si avant ce recuit, le frottement intérieur mesuré dans le même domaine de température est associé ou non au pic $\mathrm{P}_{2}$ (Fig. 2 courbes 4 , $5,6)$. Toutefois, le fait d'observer, dès les premières montées en température, un épaulement vers $280 \mathrm{~K}$ semblant lié au pic $P_{2}$, nous incite à penser que le frottement intérieur mesuré entre l'ambiante et $400 \mathrm{~K}$ serait associé au pic $P_{2}$. Il convient tout de même d'être prudent car on ne peut exclure définitivement la présence d'un fond continu surtout après recuit au-dessus de $550 \mathrm{~K}$. La décroissance du frottement intérieur mesuré à $293 \mathrm{~K}$ traduit donc probablement l'évolution de la hauteur de $\mathrm{P}_{2}$ : cette décroissance est maximale vers 500-550 $\mathrm{K}$ (comme le montre la courbe dérivée $1^{\prime}$ Fig. 4). Cette décroissance ne peut être due qu'à un blocage des dislocations. Actuellement, il n'est pas aisé d'identifier la nature des défauts épinglant les dislocations; nous pouvons toutefois remarquer que les résultats obtenus par Chen et al. [11] après irradiation indiquent que des défauts de type interstitiel migrent dans ce domaine de température. Ainsi, l'hypothèse d'une migration des défauts interstitiels entre 470 et $670 \mathrm{~K}$ environ vers les dislocations responsables du frottement intérieur est plausible, d'autant plus que certains auteurs $[12,13]$ ont bien mis en évidence la création de défauts interstitiels par déformation plastique dans les cristaux ioniques.

L'effet des impuretés ne doit pas être pour autant négligé et notamment l'effet de la valence du fer. Cet effet sera étudié ultérieurement et en particulier il serait intéressant de pouvoir disposer de monocristaux de plus grande pureté de dimensions appropriées.

L'énergie d'activation de $P_{2}$ (estimée à $0,7 \mathrm{eV}$ environ) correspond à la barrière d'activation pour la formation de doubles décrochements sur les dislocations vis $[7,8]$ : le pic $P_{2}$ pourrait donc être identifié à un pic de Bordoni. Dans cette hypothèse, l'épaulement observé vers $280 \mathrm{~K}$ pourrait correspondre au pic de Bordoni associé aux dislocations coins, l'énergie d'activation étant plus faible pour ce type de dislocations. On observe d'ailleurs que cet épaulement se recuit plus vite que le pic $\mathrm{P}_{2}$, ce comportement étant semblable à celui des métaux.

Les pics $\mathbf{P}_{3}, \mathbf{P}_{4}, \mathbf{P}_{5}$ (de faible hauteur) apparaissent après le recuit quasi total du pic $P_{2}$ (apparition de $P_{3}$ ) et du pic $P_{1}$ (apparition simultanée de $P_{4}$ et $P_{5}$ ). Les pics $P_{1}$ et $P_{2}$ ayant été attribués aux propriétés intrinsèques des dislocations et les recuits de $\mathrm{P}_{1}, \mathrm{P}_{2}$ à l'épinglage des dislocations, il est suggéré que ces pics $\mathrm{P}_{3}, \mathrm{P}_{4}$ et $\mathrm{P}_{5}$ pourraient être dus à l'interaction entre dislocations et défauts ponctuels. Ces pics étant analogues aux pics de Hasiguti [14] observés dans les métaux. Le pic $P_{3}$ serait ainsi dû à l'interaction défauts ponctuels-segments de dislocation reposant dans les vallées de Peierls, les pics $\mathrm{P}_{4}$ et $P_{5}$ résulteraient de l'interaction défauts ponctuelsdécrochements géométriques. Il est évident que cette hypothèse reste à confirmer par des études complémentaires.

\section{Bibliographie}

[1] De Batist, R., Defects and Their Structure in Nonmetallic Solids edited by B. Henderson and A. E. Hughes (Plenum Press) 1975.

[2] Southgate, P. D., Mendelson, K. S., De Perro, P. L., J. Appl. Phys. 37 (1966) 206.

[3] Dahlberg, P., Carnahan, R. D., Brittain, J. O., J. Appl. Phys. 33 (1962) 3493.

[4] Ritchie, I. G., Sprungmann, K. W., Script. Met. 7 (1973) 323.

[5] Chang, R., J. Appl. Phys. 32 (1961) 1127.

[6] Ikushima, A., Suzuki, T.. J. Phys. Soc. Jpn 18 (1963), suppl. I, 163.

[7] Woo, C. H., Puls, M. P., Philos. Mag. 35 (1977) 3, 727.
[8] Singh, R. N., Coble, R. L., J. Appl. Phys. 45 (1974) 981995.

[9] Turner, T. J., Murphy, C., Schultheiss, T., Phys. Status Solidi (b) 58 (1973) 843.

[10] Sibley, W. A., Kolopus, J. L., Mallard, W. C., Phys. Status Solidi 31 (1969) 223.

[11] Chen, Y., Williams, R. T., Sibley, W. A., Phys. Rev. 182 (1969) 3960

[12] Boni, M., Camagni, P., Colour Centres in Ionic Crystals, Reading (1971) H154.

[13] Clark, C. D., Hawkins, J., Newman, D. H., Colour Centres in Ionic Crystals, Reading (1971) H155.

[14] Nowick, A. S. et Berry, B. S., Anelastic Relaxation in Crystalline Solids (Academic Press, New York) 1972. 